\title{
Appropriate care for older people with cognitive impairment in hospital
}

\author{
Gideon A Caplan ${ }^{1,2}$, Susan E Kurrle ${ }^{3,4}$, Anne Cumming
}

$\mathrm{M}$ ore than half of the patients in adult hospitals are over 65 years of age, many of them frail with multiple comorbidities, frequently including some degree of cognitive impairment. The most common "side effect" experienced by older people in hospital is delirium, sometimes accompanying the illness that brought them to hospital (prevalent delirium), but too frequently developing in hospital (incident delirium) due to misadventure, illnesses contracted, and/or treatments and procedures administered.

Delirium is an acute syndrome characterised by altered levels of consciousness, attention and cognitive function. The overall occurrence of delirium, including prevalent and incident delirium, ranges from $29 \%$ to $64 \%$ on general or geriatric medicine wards, $11 \%$ to $68 \%$ on surgical wards (highest in orthopaedics), and $26 \%$ to $82 \%$ in intensive care units (ICUs). However, many studies exclude people with dementia, thereby likely underestimating the true rates. ${ }^{1}$ These rates are only found when delirium is actively and frequently screened for by trained researchers. In practice, delirium is missed at least $50 \%$ of the time. ${ }^{2,3}$ The reason that poor detection is of such concern is that delirium is a medical emergency that leads to a $50-500 \%$ increase in mortality, with greater disability, cognitive impairment and rates of institutionalisation among survivors. ${ }^{1}$

\section{Delirium prevention}

It is important to remember that delirium can be prevented. The Hospital Elder Life Program (HELP) study found that a multicomponent intervention aimed at minimising six risk factors for delirium (cognitive impairment, sleep deprivation, immobility, visual impairment, hearing impairment, dehydration) reduced the odds of developing delirium by $40 \%$ in medical patients, as well as reducing the days with delirium and the number of episodes. ${ }^{4}$ A similar intervention has proven effectiveness in Australia. ${ }^{5}$ A recent review of 11 non-pharmacological intervention studies found a $53 \%$ reduction in delirium and $62 \%$ reduction in falls. ${ }^{6}$

A randomised controlled trial of delirium prevention in older orthopaedic patients found that geriatric consultation preoperatively or within 24 hours postoperatively, along with a geriatrician visiting daily in hospital making targeted recommendations based on a structured protocol, reduced delirium by $36 \%$, with a particular reduction in severe delirium. ${ }^{7}$ Hospital in the Home treatment is another option that reduces the incidence of delirium and mortality. 8,9

\section{Screening for delirium}

Because delirium can either be found on presentation to the emergency department (ED) or develop in hospital, screening should occur throughout the admission, particularly in high risk contexts such as the ED or ICU, on the orthopaedic or geriatric

\section{Summary}

- More than half of the patients in adult hospitals are over 65 years of age. Although not a normal part of ageing, many older people will present to hospital with cognitive impairment (dementia or delirium) along with other complex comorbidities.

- Older people, and particularly those with dementia, are also at increased risk of developing delirium during their hospital stay. Delirium has serious short and long term consequences, such as increased mortality, falls, accelerated functional and cognitive decline, and earlier entry to residential care.

- Appropriate delirium care consists of introducing evidencebased prevention strategies for all patients at risk. For patients with delirium, it is crucial that delirium is not missed and that the underlying causes are identified and treated. Screening, assessment and a systematic workup is vital. As well as treating the underlying medical and surgical causes, the involvement of family members and a calm, safe environment are important.

- Patients with cognitive impairment should receive person-centred, goal-directed care so that their particular risks of harm are identified and minimised, and their care is aligned with their preferences and is medically appropriate for their circumstances.

- Three de-identified, composite case scenarios illustrate, respectively, the role of medicines in causing delirium, how family members can assist in evaluation, and the importance of the appropriate management of post-operative delirium.

medicine wards, or after any surgical procedure. Delirium can affect people at any age, but the risk is highest and the consequences are greatest for older people. Many useful screening tools have been developed. These include the Abbreviated Mental Test Score, which screens for cognitive impairment, ${ }^{10}$ or the 4AT, a rapid assessment test for delirium and cognitive impairment, ${ }^{11}$ and the 7-second RADAR (Recognizing acute delirium as part of your routine) screening tool, which is incorporated into the medication round. ${ }^{12}$ Delirium has different subtypes: hypoactive, hyperactive and mixed. The hyperactive or agitated subtype draws attention to itself, whereas the hypoactive subtype, sometimes described as "quietly confused", is often mistaken for dementia or depression.

Screening should be done as part of a stepped approach to diagnosis and management to support frontline ward staff to care for their patients effectively. Patients who screen positive for delirium require a diagnostic test and then a workup. A number of diagnostic tools are available. One such tool is the Confusion Assessment Method, which also has a version for use in the ICU. ${ }^{13,14}$ Such diagnostic tests are aided by consultation with the family or carers, checking to see if the patient seems different to normal. Screening and diagnosis are an important part of the comprehensive geriatric assessment process. 


\section{Considering dementia}

Many patients in hospital have cognitive impairment due to dementia, but without delirium. Dementia is a major risk factor for delirium, so it is very important to check for delirium in a person with dementia, but not everyone with dementia will develop delirium in hospital. People with dementia in hospital still require action to prevent them developing delirium. Half will experience a deterioration in their dementia as a result of that hospitalisation with an increased risk of requiring admission to a residential aged care facility - so person-centred care designed to meet their particular needs is crucial. This care needs to include a multicomponent intervention to prevent delirium, while also aiming to prevent pressure sores, dehydration, functional decline and stress. ${ }^{15}$

Appropriate care in hospital for people with dementia needs to take account of the stage of their dementia. Early dementia in a person living at home with independence in most activities of daily living (ADLs) and a good quality of life should not exclude a wide range of therapeutic interventions.

However, end-stage dementia where someone is bed-bound, at times with contractures, very limited or no vocabulary and experiencing episodes of aspiration or dehydration due to poor oral intake, indicates that many therapeutic options are futile. In this situation, there is no evidence to suggest that feeding tubes will improve longevity, cause weight gain or prevent aspiration or pressure sores. $^{16}$

\section{Management - evaluation}

Once a diagnosis of delirium is made, it is imperative to evaluate the patient for predisposing conditions (vulnerability) and precipitating causes or noxious insults, before admission or in hospital, which are potentially treatable. The more vulnerable or frail the patient, the smaller or more benign the putative insult that can trigger delirium. ${ }^{2}$ Many, if not most, patients with delirium will have difficulty giving a clear history, although extremely useful information can still be obtained, especially with a corroborative history from the family, carer or general practitioner. A comprehensive assessment and systematic workup is mandatory. Older patients have a greater need for diagnostic tests because of their atypical presentation and difficulty accurately reporting their symptoms while acutely confused, and higher incidence of a wide range of conditions.

Patients with delirium often have a multiplicity of identifiable pathology and drugs that may be triggering delirium. Any centrally acting drugs or drugs with anticholinergic properties are particularly suspect (see the case scenario in Box 1). ${ }^{17}$ Judicious multifaceted intervention is therefore required in an attempt to restore homoeostasis, including withdrawal of aggravating medications. For example, when unable to distinguish between pulmonary oedema and chest infection, clinicians can treat both conditions, even if this involves the seemingly contradictory notion of intravenous frusemide with gentle intravenous rehydration. Frail older people are relatively immunocompromised and may not display the full symptoms and signs of infection, or other conditions, which healthy younger people do. Delayed treatment, waiting for an illness to fully declare itself, is generally the worst option. However, part of the evaluation of a patient with delirium should include discussion with the family about the goals of treatment. Burdensome treatment at the end of life should be avoided, and the broader use of advance care planning greatly assists with communicating which options are preferred. ${ }^{18}$

The nature of delirium is that it does not follow office hours, often presenting in the middle of the night (see the case scenario in Box 2).
1 Case scenario: can medicine to help you walk also help you fall?

$\mathrm{Mr} \mathrm{B}$ is an 82-year-old man living alone in his home since the death of his wife 3 years earlier. He was brought into the emergency department by ambulance after having fallen over in his local shopping centre. The ambulance report noted that he appeared confused and there was concern that he may have hit his head. Mr B's confusion worsened and he required sedation to allow a cerebral computed tomography (CT) scan to be performed.

It took several hours for his GP to be identified through prescriptions found in his wallet. It appeared from these that he had hypertension. His GP confirmed that he had been treating him for hypertension for some years, and he had recently commenced treatment for Parkinson disease by a neurologist.

Mr B's CT scan showed cerebral atrophy consistent with his age but no recent evidence of injury. Other laboratory investigations were within normal limits. Mr B's pharmacist was contacted by staff and confirmed that, the previous week, he had dispensed Sinemet CR 200/50 (levodopa/carbidopa) three times a day.

Mr B remained agitated and a diagnosis of delirium was made. He was admitted and initially commenced on a low dose of haloperidol before the information about possible Parkinson disease was known. This resulted in significant sedation, and all medication was then withheld.

Mr B improved both cognitively and physically over the following 3 days with regular fluids and mobilisation. As it was felt that his delirium had not completely resolved, he was transferred to the rehabilitation ward for further mobilisation and assessment of his possible Parkinson disease. The cause of his delirium was felt most likely to be the relatively high dose of Sinemet that he had been taking for the several days before admission.

Therefore, all staff need to have some familiarity with delirium recognition and management and access to resources on the ward. ${ }^{19}$ Hospitals need to have multicomponent support to assist with more complex cases of delirium to improve outcomes; ${ }^{6,7}$ such support includes geriatric or psychogeriatric consultation, hopefully with both nursing and medical experts, and ideally a delirium unit for complex behavioural issues. ${ }^{20}$ Physical and chemical restraints will frequently exacerbate delirium and lead to worse injuries - delirium is one of the most common causes of falls in hospital (see the case scenario in Box 3) - as well as causing a permanent deterioration in cognitive function, accelerating the rate

\section{Case scenario: can delirium occur before the age of 65 ?}

Mrs S is a 62-year-old lady with younger onset Alzheimer disease. She is otherwise physically well and lives with her husband in their own home, with support from their daughter who lives nearby. Mrs S requires prompting or assistance with most activities of daily living but can still toilet herself independently and feed herself if food is provided for her.

One evening, she became very confused, constantly repeating herself and walking around the house looking for her husband who had gone out. She could not be settled and her daughter rang the local GP and then an ambulance. In hospital, Mrs S became very agitated, screaming for the police and becoming very aggressive to staff when they tried to examine her and take blood.

Mrs S's daughter had accompanied her mother and explained to the emergency department staff that her mother had moderate dementia but was normally quite calm and cooperative and that this behaviour was a dramatic change. ED staff placed Mrs $S$ in a quiet room with her daughter until the results of investigations indicated that Mrs S had a urinary tract infection with elevated white cell count and C-reactive protein level. She was treated with intravenous antibiotics and fluids, and her daughter remained with her overnight.

Her agitation had decreased in the morning with the appearance of her husband, and she was discharged home with follow-up from the Hospital in the Home team to administer antibiotics and ensure adequate hydration. Mrs S's GP agreed to review her later. 


\section{Case scenario: fractured hips and delirium}

Mrs $W$ is an 84-year-old lady who lives with her husband in their own home. She has some cognitive impairment and has suspected early dementia. However, she is still quite independent in activities of daily living and is physically active. She has a known predisposition to delirium, having had an episode with a chest infection some months earlier. Her medication included regular vitamin D, salbutamol and paracetamol as needed.

Mrs W fell while vacuuming the stairs in her house and sustained a left hip fracture. She was admitted to hospital and gave a clear history in the emergency department. There was no obvious cognitive impairment noted.

Following hip fracture surgery, she spent a prolonged period in recovery and was left on her own. She became quite confused and, thinking that her husband was being taken away on a trolley, she climbed out of bed, fell, and re-fractured her hip. She was found to have a post-operative delirium, due to the anaesthetic and analgesic drugs she had been receiving. This was treated supportively with fluids and pain relief, and resolved over 2 weeks.

She required 2 months of bed rest in hospital before revision of her hip fracture was able to be performed. This was spent mainly in the rehabilitation ward, allowing her to maintain her upper limb strength and balance. Regular screening for delirium occurred during this time, and she had one brief episode due to a urinary tract infection.

Following revision of the hip replacement, she had shortening of $3 \mathrm{~cm}$ to the left leg, requiring a $3 \mathrm{~cm}$ shoe raise and a cane when mobilising. Mrs $\mathrm{W}$ was able to return home after a further period of rehabilitation but remained significantly disabled due to mobility limitation associated with her shortened limb.

of progression of cognitive impairment, and worsening ADL function. ${ }^{21}$ Therefore, prevention and early treatment of delirium has many flow-on benefits.

\section{Management - treatment}

Treatment of delirium is directed at the underlying medical and surgical causes of the syndrome. Addressing the six HELP risk factors is also useful as part of the treatment, and families can play a beneficial role in calming agitated patients and providing familiar objects from home. In some cases, an assistant-in-nursing or volunteer to sit with the patient and assist with the HELP risk factors can be of great benefit. A delirium unit or room can provide a calm, comfortable environment, which is helpful for agitated or wandering patients.

As antipsychotic medicines have only modest benefit and known serious effects, including increased mortality, their use for patients with dementia and delirium for behavioural symptoms should be reserved for those with significant distress who are at risk to themselves or others and where non-drug strategies have been unsuccessful. ${ }^{22-24}$ The principles of low dosage, close monitoring, short duration and discussion with the patient, carers and family should be followed. If drugs are needed for severe agitation or psychosis, low dose antipsychotics are generally first line, except in delirium tremens or delirium in Lewy body dementia. We do not have any known disease-specific or disease-modifying treatments for delirium, because so little is known about its pathophysiology. Research is pointing to roles for inflammation, metabolic changes, neurotransmitter disturbance and reduced cerebral blood flow, which exacerbate many of the known features of dementia in the central nervous system. ${ }^{25-28}$ Given that delirium increases the risk of developing dementia and accelerates its progress, delirium may be best understood as the acute manifestation or exacerbation of the chronic disease, dementia; effective communication to the GP and other care providers is therefore vital.

This suggests that effective treatment of delirium will reduce the complications of delirium, including reducing mortality and placement in residential aged care; it can also be disease-modifying for dementia. Therefore, action to improve delirium prevention, recognition and management should be a priority for all (adult) hospitals.

\section{Australian Commission on Safety and Quality in Health Care resources - a better way to care}

The Australian Commission on Safety and Quality in Health Care (the Commission) has released A better way to care: safe and highquality care for patients with cognitive impairment (dementia and delirium) in hospital — a set of resources for clinicians, health service managers and consumers to improve the early recognition of, and response to, patients with cognitive impairment so that they receive safe and high quality care. ${ }^{29-31}$ These detailed resources complement and expand upon the general principles outlined in this article.

The Commission has also released the Delirium Clinical Care Standard, ${ }^{32}$ which consists of key quality statements that describe high priority areas for improvement in delirium prevention, recognition and treatment. The standard also includes suggested indicators to assist local health services to monitor implementation.

The Commission has also included the quality and safety risks for people with cognitive impairment in draft version 2 of the National Safety and Quality Health Service (NSQHS) Standards. Following their release in 2017, implementation of the revised NSQHS Standards is expected to begin from January 2019.

\begin{abstract}
Competing interests: Gideon Caplan is President of the Australasian Delirium Association and Co-Chair of the Australian Commission on Safety and Quality in Health Care Delirium Clinical Care Standard Topic Working Group; he has received grant support for research into the pathophysiology of delirium. Susan Kurrle is the Director and Chief Investigator of the National Health and Medical Research Council (NHMRC) Partnership Centre on Dealing with Cognitive and Related Functional Decline in Older People, and part of her salary is funded by the NHMRC to conduct research into cognitive decline, including delirium.
\end{abstract}

Provenance: Commissioned; externally peer reviewed.

(c) 2016 AMPCo Pty Ltd. Produced with Elsevier B.V. All rights reserved.
1 Inouye SK, Westendorp RGJ, Saczynski JS. Delirium in elderly people. Lancet 2014; 383: 911-922.

2 Caplan G. Managing delirium in older patients. Aust Prescr 2011; 34: 16-18.

3 Mistarz R, Eliott S, Whitfield A, Ernest D. Bedside nursepatient interactions do not reliably detect delirium: An observational study. Aust Crit Care 2011; 24: 126-132.

4 Inouye SK, Bogardus ST, Charpentier PA, et al. A multicomponent intervention to prevent delirium in hospitalized older patients. N Engl J Med 1999; 340: 669-676.

5 Caplan GA, Harper E. Recruitment of volunteers to improve vitality in the elderly (The REVIVE Study). Int Med J 2007; 37: 95-100.

6 Hshieh TT, Yue J, Oh E, et al. Effectiveness of multicomponent nonpharmacological delirium interventions: A meta-analysis. JAMA Int Med 2015; 175: 512-520
7 Marcantonio ER, Flacker JM, Wright J, Resnick NM. Reducing delirium after hip fracture: a randomized trial. J Am Geriatr Soc 2001; 49: 516-522.

8 Caplan GA, Coconis J, Board N, et al. Does home treatment affect delirium? A randomised controlled trial of rehabilitation of elderly and care at home or usual treatment (The REACH OUT Trial). Age Ageing 2006; 35: 53-60.

9 Caplan GA, Sulaiman NS, Mangin D, et al. A meta-analysis of Hospital in the Home. Med J Aust 
2012; 197: 512-519. https://www.mja.com.au/journal/ 2012/197/9/meta-analysis-hospital-home

10 Hodkinson HM. Evaluation of a mental test score for assessment of mental impairment in the elderly. Age Ageing 1972; 1: 233-238.

11 Bellelli G, Morandi A, Davis DHJ, et al. Validation of the 4AT, a new instrument for rapid delirium screening: a study in 234 hospitalised older people. Age Ageing 2014 43: 496-502.

12 Voyer P, Champoux N, Desrosiers J, et al. Recognizing acute delirium as part of your routine [RADAR]: a validation study. BMC Nurs 2015; 14: 19.

13 Inouye SK, Van Dyck CH, Alessi CA, et al. Clarifying confusion: the confusion assessment method. A new method for detection of delirium. Ann Intern Med 1990; 113: 941-948.

14 Ely EW, Inouye SK, Bernard GR, et al. Delirium in mechanically ventilated patients: validity and reliability of the confusion assessment method for the intensive care unit (CAM-ICU). JAMA 2001; 286 2703-2710.

15 Alzheimer's Society, Counting the cost: caring for people with dementia on hospital wards. London: Alzheimer's Society, 2009. http://www.alzheimers.org.uk/site/ scripts/documents_info.php?documentID=1199 (accessed Oct 2016)

16 Goldberg LS, Altman KW. The role of gastrostomy tube placement in advanced dementia with dysphagia: a critical review. Clin Interv Aging 2014; 9 : 1733-1739.
17 Alagiakrishnan K, Wiens CA. An approach to drug induced delirium in the elderly. Postgrad Med J 2004; 80: 388-393.

18 Caplan GA, Meller A, Chan S, et al. Advance care planning and hospital in the nursing home. Age Ageing 2006; 35: 581-585.

19 Kurrle SE, Cameron ID, Geeves RB. A quick ward assessment of older patients by junior doctors. BMJ 2015; 350: h607.

20 Wong Tin Niam DM, Geddes JA, Inderjeeth CA Delirium unit: our experience. Australas J Ageing 2009; 28: $206-210$.

21 Gross AL, Jones RN, Habtemariam DA, et al. Delirium and long-term cognitive trajectory among persons with dementia. Arch Int Med 2012; 172: 1324-1331.

22 Kalisvaart KJ, de Jonghe JFM, Bogaards MJ, et al. Haloperidol prophylaxis for elderly hip-surgery patients at risk for delirium: a randomized placebo-controlled study. J Am Geriatr Soc 2005; 53: 1658-1666.

23 Schneider LS, Dagerman KS, Insel P. Risk of death with atypical antipsychotic drug treatment for dementia: meta-analysis of randomized placebo-controlled trials. JAMA 2005; 294: 1934-1943.

24 Agar M, Lawlor P, Quinn S, et al. Phase III randomized double-blind controlled trial of oral risperidone, haloperidol or placebo with rescue subcutaneous midazolam for delirium management in palliative care. J Am Geriatr Soc 2015; 63: S99.

25 Van Gool WA, van de Beek D, Eikelenboom P. Systemic infection and delirium: when cytokines and acetylcholine collide. Lancet 2010; 375: 773-775.
26 Caplan GA, Kvelde T, Lai C, et al. Cerebrospinal fluid in long lasting delirium compared to Alzheimer's dementia. J Gerontol A Biol Sci Med Sci 2010; 65: $1130-1136$.

27 Koponen HJ. Neurochemistry and delirium. Dement Geriatr Cogn Disord 1999; 10: 339-341.

28 Caplan GA, Lan Z, Newton L, et al. Transcranial Doppler to measure cerebral blood flow in delirium superimposed on dementia. A cohort study. J Am Med Dir Assoc 2014; 15: 355-360.

29 Australian Commission on Safety and Quality in Health Care. A better way to care: Safe and high-quality care for patients with cognitive impairment (dementia and delirium) in hospital. Actions for health service managers. Sydney; ACSQHC, 2014.

30 Australian Commission on Safety and Quality in Health Care. A better way to care: Safe and high-quality care for patients with cognitive impairment (dementia and delirium) in hospital. Actions for clinicians. Sydney; ACSQHC, 2014

31 Australian Commission on Safety and Quality in Health Care. A better way to care: Safe and high-quality care for patients with cognitive impairment (dementia and delirium) in hospital. Actions for consumers. Sydney; ACSQHC, 2014

32 Australian Commission on Safety and Quality in Health Care. Delirium Clinical Care Standard. Sydney: ACSOHC, 2016. https://www.safetyandquality.gov.au/our-work/ clinical-care-standards/delirium-clinical-care-standard (accessed Oct 2016) 